\title{
Coronavirus disease 2019 (COVID-19) and physicians with a disability: a compounding stressor
}

\author{
Rama S. Ayyala ${ }^{1,2}$
}

Received: 5 November 2021 / Revised: 5 November 2021 / Accepted: 28 November 2021 / Published online: 24 January 2022 (c) The Author(s), under exclusive licence to Springer-Verlag GmbH Germany, part of Springer Nature 2021

"Fear. Stress. Anxiety. Shame. Guilt. These emotions were heightened in many due to COVID-19. For those with underlying health conditions and disabilities, the impact was even greater, with implications on personal and professional burnout."

I have had rheumatoid arthritis (RA) for 11 years. After my diagnosis during my intern year, I started medication and went on with residency as if nothing had changed. It was more important for me to excel and not let my colleagues down. During the course of my training, I sought medical care as needed, took medications to keep my RA at bay, and only shared my health issues with co-workers if flares prevented me from being able to perform procedures. When this did happen, I felt like a failure and doubted that I was a capable physician. I was convinced my co-workers would be resentful because I would be "dumping" work on them. Instead, my colleagues and attending physicians were supportive and helped me navigate my schedule appropriately, and no one ever told me that they were resentful; nevertheless, the thought always lingered in my mind.

For years, I have had intermittent flares of my disease - to the point where I am unable to move my hands. If it didn't interfere with my work, I just suffered in silence. The moments when I had to lean on my colleagues were a struggle. While asking for help is something I always emphasize to trainees, when it came to me, I saw it as a sign of weakness and as a burden to my colleagues. In the back of my head, I also struggled with the question of whether I could continue in my career path - how was I supposed to be an academic educator in pediatric radiology if there were days I couldn't comb my hair?

Rama S. Ayyala

Rama.ayyala@cchmc.org

1 Department of Radiology, Cincinnati Children's Hospital Medical Center, 3333 Burnett Ave., Cincinnati, OH 45229, USA

2 Department of Radiology, University of Cincinnati College of Medicine, Cincinnati, OH, USA
In March 2020, the world changed with the arrival of the severe acute respiratory virus 2 (SARS-CoV-2), the virus responsible for the coronavirus disease 2019 (COVID-19) pandemic. Many businesses closed, schools pivoted to distance learning, and hospitals scrambled to create optimal pathways to provide safe patient care while protecting their staff. March 2020 was also the month I had to suddenly face being honest and open about my own vulnerability. I had been immunosuppressed for nearly a decade before this pandemic, with minimal daily risk to my health. Now, I had to question almost every decision I made, both at work and outside work, and rate the relative risk to my health. This created a new constant anxiety in my head. Compounding this burden was the uncertainty of the virus and the fear of sharing my status with others - and how it would impact my capabilities of performing my job, my relationships with my colleagues, and my overall career.

I was working in a small pediatric radiology group at a hospital seeing increasing cases of COVID-19 daily. I would have to be in direct patient contact daily for US and fluoroscopy studies, especially on call, with no guarantee of having appropriate personal protective equipment. At that time, our hospital did not have enough N95 masks for its frontline workers, let alone radiologists. The feelings of inadequacy flooded my brain. I had to tell my group about my situation. It was one of the most difficult decisions and hardest conversations to have. I spent many sleepless nights thinking about how I would say what I wanted and needed to say. No matter how I tried, it always seemed to sound like: "Hey, I am one of six pediatric radiologists in our group, but I cannot come to work because I am immunocompromised and scared of dying from COVID. Good luck trying to navigate this pandemic with one less body in the reading room and call pool!" That is not exactly how the conversation went, but that is how it felt to me. In reality, I texted all my colleagues to explain the situation; all of them were extremely understanding and willing to help out. I was so lucky to have fantastic co-workers and leadership, who immediately arranged for me to work remotely from home. 
Until COVID hit, I only had to battle the challenges associated with RA. However, since then, there has been a new layer of anxiety related to my immunocompromised state. My story is only one of likely many such stories during the early phases of the COVID-19 pandemic. Disability is defined as a mental or physical impairment that limits one or more major life activities [1]. One study in 2019 showed that $4.6 \%$ of students in Doctor of Medicine programs in the United States self-reported a disability to their institutions [2]. There is a paucity of data on the number of trainees and physicians with disabilities in medicine; however, one study estimated $2 \%$ to $10 \%$ of practicing physicians develop disabilities [3]. One form of disability that is typically invisible is immunosuppression, which can be caused by a disease, or medications used to treat a disease. One study estimated approximately 3.6 million Americans are immunocompromised; however, this was thought to be an underestimate, with more accurate numbers being as high as 15 million people $[4,5]$. Given the large group of people impacted by disabilities of all types, it is a topic that deserves attention to ensure inclusion and accessibility for all in the workplace. Work is being done in medical schools and residencies to recruit and provide equal access and opportunities for those with disabilities [6]. However, there is little formal literature regarding practicing physicians with disabilities. A critical assessment of disabilities among physicians is needed, utilizing the experiences of individual physicians, to educate others and develop ways to create inclusive, Americans with Disabilities Act (ADA)-compliant work environments [7, 8].

As a radiologist, my experience during the early COVID19 pandemic included a months-long period of remotely working at home alone, with a lot of time to ruminate. So many emotions and questions plagued me. Fear, anger, sadness, hopelessness. How can I continue to be a physician safely? How do I navigate my career in ways that do not burden my colleagues in the future? Is it even possible, or would it be easier to just walk away from medicine and do a desk job where I would never have to deal with this again? How can I be a competent pediatric radiologist in the future after months of reading remotely and not seeing patients, not interacting with trainees and colleagues? To complicate matters, all this immediately preceded my transition to another pediatric radiology job at a large academic pediatric hospital in the summer of 2021. Before I even began my new job, I had to tell my new chief all of my issues and concerns. One of the biggest challenges in my journey has been being honest and vulnerable to leadership, and fearing the response. Again, I was met with unconditional support and accommodation for me to safely do my new job.

In medicine, physicians are portrayed as "heroes" - a term that conjures imagery of invincibility. There is an unsaid rule that everyone needs to remain strong and not show weakness, making it difficult for one to be truly honest and accepting of one's shortcomings or limitations. It is difficult to ask for help without feeling inadequate. It is difficult to admit being incapable of performing certain duties, for fear of seeming incompetent or putting more work on others. It is difficult to say no to professional obligations or opportunities, such as traveling in-person for lectures and conferences, because of perceived high personal risk. Living with a disability is physically and emotionally draining, with the COVID-19 pandemic making it even more challenging. I have spent years doing research and writing about burnout in medicine, but my experiences during COVID highlighted a new aspect of my burnout. Being unable to fulfill one's job to the fullest extent increases emotional exhaustion and perception of increased lack of accomplishment. The anger associated with feeling inadequate can instill callous attitudes toward the job, especially because the medical culture has been deeply rooted in physicians hiding weaknesses. This was all compounding my professional burnout.

Recently, as I have been reflecting on my experiences during this time, I realized that many others are struggling, such as those with underlying health constraints and those with close family members deemed to be at high risk for COVID19. Aside from the situations heightened by COVID-19, I thought more deeply about the many who had disabilities and personal health situations pre-COVID that they have navigated silently in the past. I have learned some lessons with my experience that I hope help others in similar situations. I also believe this is an opportunity to raise awareness of those with all types of disabilities practicing medicine, and to strive to create more inclusive and accessible work environments.

Over the last 2 years, one vital factor that helped me has been the support of my colleagues, family and friends. The power of community has never been more heightened to me. I have been fortunate to have leadership and colleagues at my institutions who have understood my situation, made accommodations and supported me extensively during this period. However, many with disabilities in medicine might not be as fortunate. Full access for those with disabilities is crucial and might require removal of environmental, physical or social barriers. Creating accommodations does not have to be disruptive and expensive - it can be as simple as listening to colleagues and helping one another out (i.e. covering shifts, providing social support). A culture of safety and fostering a personal sense of psychological safety at work is critical to helping those affected feel included and accepted. Stigmatization of these issues needs to be abolished in medicine, and this begins at the institution and leadership levels. Fostering honest conversations about these issues will help others like me feel more comfortable with being vulnerable and open, creating a better work culture and environment [9]. 
The advent of virtual conference capabilities has allowed me to continue to be active with my academic aspirations; however, as we plan for the future beyond the COVID-19 pandemic, I have been faced with choosing my health over participating in academic conferences and talks because of the expectation that we will be returning to pre-pandemic norms of in-person conferences. The loss of these opportunities has been extremely challenging and frustrating. Many others in medicine have similar concerns, either because of risks associated with personal health or risks to loved ones or household members. This is especially true at the time of this writing. There is still no U.S. Food and Drug Administration (FDA)-approved COVID vaccine for children younger than 5 years, and pediatric cases are rising. Given the overall change in our society during the last 2 years, and the uncertainty of when this will stabilize, we need to formulate a future with more accommodations for everyone to participate in work-related events safely, including more virtual and hybrid options. Similarly, flexible work schedules and environments will be important to incorporate into departments and institutions to accommodate vulnerable individuals if future situations similar to the COVID-19 pandemic arise.

Being open and honest about one's personal struggles is scary. However, being open and honest normalizes the associated stress and anxiety, which helps others: this means seeing that others are suffering like ourselves, that not everyone is perfect, and that we can all lean on one other for help. Many of us are going through significant life stresses and changes, and everyone has different mechanisms to cope. Some might be going through new challenges, especially in the time of COVID-19, and suddenly faced with uncertainty and loss of ways to cope. Here are some potential areas of focus to help all of us. We can:

- Create awareness about physicians with disabilities to encourage individuals to be open and honest about stresses and anxieties associated with personal health issues or disabilities.

- Ensure safe and inclusive work environments that accommodate those who need it without stigmatization or repercussions.

- Address system and culture changes that can foster psychological safety in the workplace to minimize work- related stress adding to the existing stresses and anxieties.

I am fortunate to be a part of an amazing pediatric radiology community, with supportive colleagues, leaders, mentors and friends. My purpose in sharing my experience is to highlight different areas where we can strive to improve and learn as a community. These last 2 years have been difficult for all of us in different ways - many people have similar experiences and stories to share. My hope is that we can learn from one another and help uplift one another, and that our community will lead by example.

\section{References}

1. Feldblum CR, Barry K, Benfer EA (2008) The ADA Amendments Act of 2008. Tex J CL CR

2. Meeks LM, Case B, Herzer K et al (2019) Change in prevalence of disabilities and accommodation practices among U.S. medical schools, 2016 vs. 2019. JAMA 322:2022-2024

3. DeLisa JA, Thomas P (2005) Physicians with disabilities and the physician workforce: a need to reassess our policies. Am J Phys Med Rehabil 84:5-11

4. Wallace BI, Kenney B, Malani PN et al (2021) Prevalence of immunosuppressive drug use among commercially insured US adults, 2018-2019. JAMA Netw Open 4:e214920

5. Requarth T (2021) 'What exactly is the plan for us?': millions of immunocompromised people, left behind by the vaccine, are facing a stark future. Slate. https://slate.com/technology/2021/10/ immunocompromised-covid-risk-delta-vaccine-response.html. Accessed 19 Oct 2021

6. Meeks LM, Jain NR, Moreland C et al (2019) Realizing a diverse and inclusive workforce: equal access for residents with disabilities. J Grad Med Educ 11:498-503

7. Meeks LM, Herzer K, Jain NR (2018) Removing barriers and facilitating access: increasing the number of physicians with disabilities. Acad Med 93:540-543

8. Schwarz CM, Zetkulic M (2019) You belong in the room: addressing the underrepresentation of physicians with physical disabilities. Acad Med 94:17-19

9. Davis P (2021) Beating burnout at work: why teams hold the secret to well-being and resilience. University of Pennsylvania Press, Philadelphia

Publisher's note Springer Nature remains neutral with regard to jurisdictional claims in published maps and institutional affiliations. 